\title{
Vaporization of Strontium Atom and Ion from Strontium Oxide Applied on Tungsten and Rhenium
}

\author{
Mitsuru Asano *, Kenji Kubo* and Saburo Magari * \\ (Received 29 September 1968)
}

\begin{abstract}
A Langmuir method has been used to determine the vapor pressure and the heats of vaporization of strontium atoms from strontium oxide applied on tungsten and rhenium surfaces. The data obtained are $\log P_{\text {atm }}=5.064-2.071 \times 10^{4} / T$ and $l_{v}^{T}=$ $4.11 \pm 0.14 \mathrm{eV}$ for tungsten, and $\log P_{\mathrm{atm}}=6.095-2.496 \times 10^{4} / T$ and $l_{u}^{T}=4.95 \pm 0.14 \mathrm{eV}$ for rhenium, respectively. By measuring the ion current of ${ }^{8} \mathrm{Sr}^{+}$with a mass spectrometer the values of the heats of vaporization of strontium ions have also been obtained as $l_{+}^{T}=7.07 \pm 0.08 \mathrm{eV}$ and $l_{+}^{T}=6.73 \pm 0.07 \mathrm{eV}$ for tungsten and rhenium, respectively, under the assumption that the ion vaporization processes may be de. scribed by the Saha-Langmuir equation. The work functions of the surfaces applied with strontium oxide have been discussed.
\end{abstract}

\section{Introduction}

The high temperature thermochemistry concerning with the vaporization and thermal dissociation processes of metallic oxides, surface ionization of atoms and thermoelectronic emission from the metallic surfaces have been intensively in. vestigated by many workers. ${ }^{1 / 7}$ The vapor pressure and the heat of vaporization of strontium oxide have been studied by a sealed tube method, ${ }^{\gamma \sim 10)}$ and the gaseous species leaving strontium oxide on metallic surfaces have also been investigated mass spectrometrically ${ }^{11 \sim 14)}$ It has been found that when strontium oxide applied on tungsten is heated, strontium oxide reacts with tungsten according to the following equation, and strontium atom vaporizes,

$$
\begin{aligned}
\frac{3}{2} \mathrm{SrO}(s)+\frac{1}{2} \mathrm{~W}(s) \rightarrow & \frac{1}{2} \mathrm{SrWO}_{3}(s) \\
& +\mathrm{Sr}(g) .
\end{aligned}
$$

But strontium oxide on platinum decom. poses predominantly to strontium and oxygen, and vaporizes according to Eq. (2),

$$
\mathrm{SrO}(s) \stackrel{\mathrm{Pt}}{\longrightarrow} \mathrm{Sr}(\mathrm{g})+\frac{1}{2} \mathrm{O}_{2} .
$$

In the studies by the sealed tube method mentioned above, however, calculations were carried out with the assumption that strontium oxide on platinum vaporizes mainly as gaseous strontium oxide molecules, not as strontium atoms.

Further, many papers dealing with the surface ionization phenomena of strontium have been published,' but the values of the heats of vaporization of strontium ions from various metallic surfaces are

* Engineering Research Institute, Kyoto University (Gokasho, Uji, Kyoto, Japan). 
little reported.

In the present work, the authors employed a Langmuir method to determine the vapor pressure of strontium atoms from strontium oxide on tungsten and rhenium, and calculated the values of the heats of vaporization of strontium atoms. The values of the heats of vaporization of strontium ions from the surfaces mentioned above were also obtained from the ion current of ${ }^{88} \mathrm{Sr}^{+}$with a mass spectrometer under the assumption that the vapor. ization processes of ions may be described by the Saha-Langmuir equation.

Then the work functions of the surfaces applied with strontium oxide were discus. sed.

\section{Experimental}

Tungsten and rhenium metals used in this work were ribbon filaments ( $\mathrm{H}$. Cross Co., USA) with a width of $0.75 \mathrm{~mm}$ and a thickness of $0.025 \mathrm{~mm}$, and were aged at about $2100^{\circ} \mathrm{C}$ for $50 \mathrm{hr}$ in vacuum.

Strontium nitrate was used to prepare the strontium oxide. A reagent grade strontium nitrate was purified by a carbonate method and crystallized out from water.

For the measurements of the vapor pressure of strontium atoms from strontium oxide applied on metals, the following procedures were used. A ribbon filament of $35 \mathrm{~mm}$ length was welded to the lead plates of stainless steel. One lead plate is screwed in a groove of the fixed leg, and another is held in a sliding groove and applied tension by a tungsten spring, by which the deformation of ribbon filament was completely eliminated. ${ }^{1(i)}$ Using a micro syringe, $1 \mu l$ of strontium nitrate solution containing $20.5 \mu \mathrm{g}$ of strontium was applied on the central part of the filament for a length of $3-4 \mathrm{~mm}$ and dried under an infra-red lamp. The filament was placed in a vacuum chamber, and then slowly heated by controlling DC current and strontium nitrate was thermally decomposed into strontium oxide. After these treatments the-temperature of filament was further raised to an experimental temperature, and maintained for a given period.

The rate of strontium atoms vaporized from strontium oxide was estimated by measuring the amount of strontium oxide remained on the filament. The filament was cut off from the lead plates and trans. fered to a $50 \mathrm{ml}$ beaker. To dissolve the strontium oxide, $1 \mathrm{ml}$ of $0.1 \mathrm{~N}$ nitric acid solution was added and warmed gently for about $4 \mathrm{~min}$. After the solution was neutralized with $0.2 \mathrm{~N}$ sodium hydroxide solution, $2 \mathrm{ml}$ of ammonium hydroxide-ammonium chloride buffer solution ( $\mathrm{PH}=9.8), 10 \mathrm{ml}$ of $0.001 \mathrm{~N}$ EDTA-0.001M magnesium sulfate solution, two drops of $5 \%$ potassium cyanide solution and two drops of BT indicator were added successively. Then the solution was titrated with $0.001 \mathrm{~N}$ EDTA solution to determing the amount of strontium oxide. The functions of magnesium sulfate and potassium cyanide are to make clear the color change at the end point of titration and to mask the interfering ions, respectively. The absolute error in this method was within $0.5 \mu \mathrm{g}$ of strontium. Since the amount of strontium remained on the filament was $7-12 \mu \mathrm{g}$ in each run, this error was never greater than $10 \%$.

A single focusing $60^{\circ}, 20 \mathrm{~cm}$ radius mass spectrometer equipped with a single filament surface ionization ion source was used for the measurements of ion current of strontium ions vaporized from strontium oxide. The filament was mounted by an improved method ${ }^{16 /}$ to eliminate its misalignment caused by thermal expansion at high temperature and to improve the 
reproducibility of ion current. The application of strontium oxide on the filament was carried out by the same procedures as for the vapor pressure measurements.

The measurement of temperature was performed by sighting with an optical pyrometer on the opposite side of the ribbon filament surface on which strontium oxide was applied, and the observed values were corrected for emissivity of the filament material ${ }^{(i)}$ and for trans. mission coefficient through the silica window of a mass spectrometer and the glass wall of a vacuum apparatus.

\section{Results and Discussions}

\section{1 Vaporization Studies of Stronti. um Atoms}

The rate of strontium atoms vaporized from strontium oxide applied on tungsten and rhenium filaments was measured at a series of temperatures and the vapor pressure was calculated at each tem. perature. If $m$ is the rate of vaporization in $\mathrm{g} / \mathrm{cm}^{2} \cdot \mathrm{sec}$, the vapor pressure can be deduced from the Langmuir equation,

$$
P=m\left(\frac{2 \pi R T}{M}\right)^{\frac{1}{2}}
$$

taking the vaporization coefficient to be unity, where $M$ is the atomic weight of strontium, $87.63, R$ is the gas constant, $8.314 \times 10^{7} \mathrm{erg} / \mathrm{deg}, P$ is the vapor pressure in dyne $/ \mathrm{cm}^{2}$ and $T$ is the absolute temperature. The results of these Langmuir studies are shown in Fig. 1. The lines plotted represent the least squares treatments of the data and follow the equations,

$$
\log P_{\mathrm{atm}}=5.064-\frac{2.071 \times 10^{4}}{T}
$$

for the vapor pressure of strontium atoms from strontium oxide on tungsten surface at the temperature range $1465^{\circ}$ to $1777^{\circ} \mathrm{K}$, and $\log P_{\mathrm{alm}}=6.095-\frac{2.496 \times 10^{4}}{T}$

for rhenium surface at the temperature range $1636^{\circ}$ to $1917^{\circ} \mathrm{K}$. And from the slopes of these lines the values of the heats of vaporization of strontium atoms were calculated as $4.11 \pm 0.14 \mathrm{eV}$ and $4.95 \pm 0.14 \mathrm{eV}$ for tungsten and rhenium, respectively, where the uncertainties given were the standard deviations for the least squares treatments. It can be considered that the behavior of rhenium upon strontium oxide is similar to that of platinum. So the data for rhenium were calculated from Eq. (2).

For the vaporization from tungsten, the vapor pressure of strontium atoms from strontium oxide has been investigated by Moore, Allison and Morrison ${ }^{(1)}$ at the temperature $1150^{\circ}$ to $1550^{\circ} \mathrm{K}$ and by Wohler and Rossini.' The values of the heats of vaporization were calculated as 3. $68 \mathrm{eV}$ by Moore, Allison and Morrison, and as $4.10 \mathrm{eV}$ by Wohler and Rossini, respectively. The value obtained in the

$T^{\circ} \mathrm{K}$

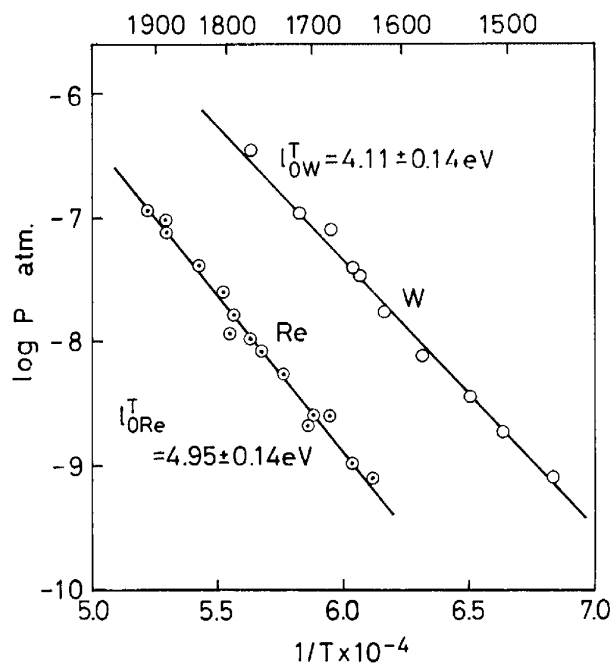

Fig. 1. Vapor pressure of strontium atoms from strontium oxide applied on tungsten and rhenium. 
present work is in poor agreement with that of Moore, Allison and Morrison, but in good agreement with that of Wohler and Rossini.

For the vaporization from platinum, behavior of which is similar to rhenium, Claassen and Veenemans ${ }^{8}$ have investigated the vapor pressure of strontium oxide at the temperature range $1500^{\circ}$ to $1650^{\circ} \mathrm{K}$ and calculated the value of the heat of vaporization as $6.12 \mathrm{eV}$ from the slope of the plot of their data with the assumption that the gaseous species is strontium oxide. Blewett ${ }^{7}$ has assumed that the difference in specific heats between gaseous and solid strontium oxide is $3 \mathrm{R}$ at all temperatures and obtained the value of the heat of vaporization at $0^{\circ} \mathrm{K}$ as $5.08 \mathrm{eV}$ from the data of Claassen and Veenemans. Moore, Allison and Struthers ${ }^{9 /}$ have measured the vapor pressure of strontium oxide at the temperature range $1290^{\circ}$ to $1650^{\circ} \mathrm{K}$, and obtained the value of the heat of vaporization at $0^{\circ} \mathrm{K}$ as $5.47 \mathrm{eV}$ with the assumption that the gaseous species is strontium oxide. However, it is mass spectrometrically believed that the strontium oxide applied on platinum decomposes predominantly to strontium and oxygen, and vaporizes as shown in Eq. (2). Namely, Aldrich ${ }^{12)}$ has observed that the ratio of $\mathrm{Sr}^{+}$ion current to $\mathrm{SrO}^{+}$is very high and the sum of $\mathrm{O}^{+}$, $\mathrm{O}_{2}^{+}$and $\mathrm{O}_{2}^{++}$ion currents is about onehalf of the $\mathrm{Sr}^{+}$ion current, and obtained the value of the heat of vaporization of strontium atoms as $6.25 \mathrm{cV}$ from the slope of the plot of his data. Bickel and Holroyd ${ }^{13)}$ have also investigated the vapor species with a mass spectrometer and observed the similar results to those of Aldrich, and obtained the value of the heat of vaporization of strontium atoms as $2.60 \mathrm{eV}$ for the higher temperature range and as $5.99 \mathrm{cV}$ for the low. er range. The values obtained by the above workers are in poor agreement with each other.

In the experiments of Claassen and Veenemans, and Moore, Allison and Struthers, the vaporization was carried out in a sealed tube in which strontium atoms vaporized from heated platinum to a cold collector wall and the atoms could easily recombine with oxygen dissociated. Thus even though the applied and deposited materials are strontium oxide, the vapor species may be strontium atoms as found by a mass spectrometer.

\section{2 Vaporization Studies of Stronti- um Ions}

The ratio of the fluxes of positive ions to atoms vaporized from a metallic surface, if they are in thermal equilibrium, is given by the Saha-Langmuir equation,

$$
\frac{N_{+}}{N_{0}}=\frac{\omega+}{\omega_{0}} \exp \left[\frac{e(\phi-I)}{k T}\right],
$$

where $N_{+}$and $N_{U}$ are the fluxes of positive ions and atoms, $\phi$ and $I$ are the work function of the surface and the first ionization potential of the atom, $\omega_{+}$and $\omega_{0}$ are the statistical weights for positive ions and atoms and $k$ is the Boltzman constant, respectively. The vapor pressure of neutral atoms is given by the following equation,

$$
P=\exp \left[\left(\frac{\Delta S^{0}}{k}\right)-\left(-\frac{l_{0}^{T}}{k T}\right)\right],
$$

and the vapor pressure is related to the rate of atoms as follows,

$$
P=N_{0}\left(2 \pi m_{\mathrm{a}} k T\right)^{\frac{1}{2}},
$$

where $\Delta S^{0}$ is the standard entropy of 


\section{Vaporizaiton of Strontium Atom and Ion from Storontium Oxide}

vaporization, $l_{0}^{T}$ is the heat of vapori. zation of atoms at temperature $T$ and $m_{\mathrm{a}}$ is the mass of the atom, respectively.

Substituting Eqs.(5) and (6) into Eq. (4), the following equation is obtained,

$$
\begin{aligned}
\mathrm{N}_{+} T^{\frac{1}{2}}= & \left(\frac{\omega_{+}}{\omega_{0}}\right) \frac{\exp \left(\Delta S^{0} / k\right)}{\left(2 \pi m_{\mathrm{a}} k\right)^{\frac{1}{2}}} \\
& \times \exp \left[\frac{\mathrm{e}(\phi-I)-l_{0}^{T}}{k T}\right] .
\end{aligned}
$$

The value of $e(\phi-I)-l_{0}^{T}$ is equal to the heat of vaporization of ions, $l^{T}$, at temperature $T$, that is,

$$
e(\phi-I)-l_{0}^{T}=-l_{+}^{T},
$$

from a consideration of the following energy cycle.

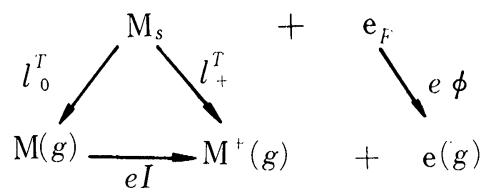

$\mathrm{M}_{s}$ is the metal atom on the surface, $\mathrm{e}_{r}$ is the electron in the Fermi level and $\mathrm{e}(\mathrm{g}), \mathrm{M}^{+}(\mathrm{g})$ are the gaseous electron, ion and atom, respectively. The values of $\Delta S^{\circ}$ and $\left(\omega_{+} / \omega_{0}\right)$ are almost unchanged over the experimental temperature range. Consequently one can estimate the value of $l^{T}$ from the slope of a straight line obtained by plotting $\log N_{+} T^{\frac{1}{2}}$ against $\mathrm{l} / T$.

Scheer and Fine have investigated the self ionization of niobium, tungsten, rhenium $^{2(1)}$ and molybdenum, and obtained the energies required for the vaporization of their positive ions using the above relations.

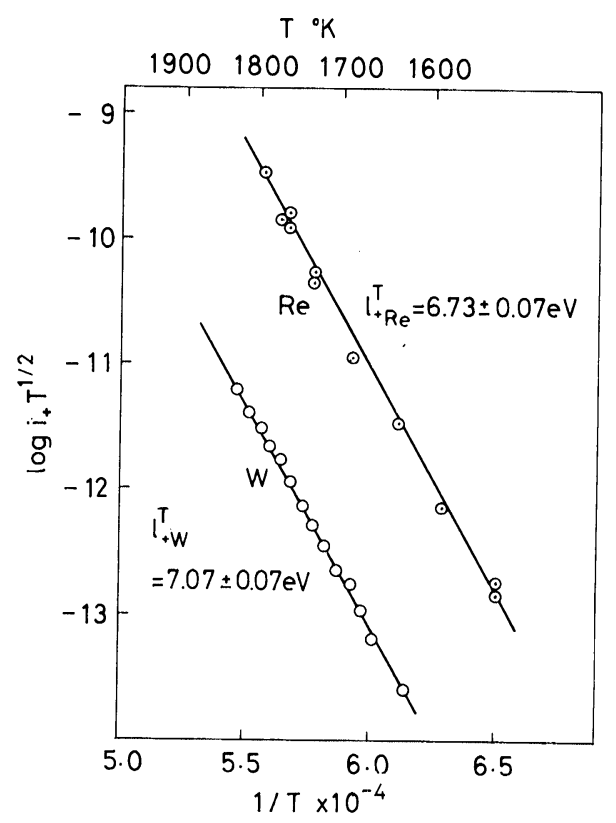

Fig. 2. $\log i_{+} \mathrm{T}^{\frac{1}{2}}$ v.s. $1 / T$.

Now, in case of the vaporization of strontium ions from strontium oxide on tungsten and rhenium, the following relation holds,

$$
N_{+} \propto i_{+},
$$

where $i_{+}$is the ion current of ${ }^{88} \mathrm{Sr}^{+}$measured by a mass spectrometer. Then, under the assumption that these processes may be

\begin{tabular}{|c|c|c|}
\hline & on tungsten & on rhenium \\
\hline$l_{0}^{1}$ & $4.11 \pm 0.14 \mathrm{eV} \quad\left(1465^{\circ}-1777^{\circ} \mathrm{K}\right)$ & $4.95 \pm 0.14 \mathrm{eV} \quad\left(1636^{\circ}-1917^{\circ} \mathrm{K}\right)$ \\
\hline$l^{1}$ & $7.07 \pm 0.08 \mathrm{eV} \quad\left(1636^{\circ}-1826^{\circ} \mathrm{K}\right)$ & $6.73 \pm 0.07 \mathrm{eV} \quad\left(1636^{\circ}-1778^{\circ} \mathrm{K}\right)$ \\
\hline$\phi$ & $2.73 \pm(1.22 \mathrm{~V}$ & $3.91 \pm 0.21 \mathrm{~V}$ \\
\hline
\end{tabular}
described by the Saha-Langmuir equation, the values of $l^{T}$ were estimated

Table 1. Values of $l_{0}^{T}, l_{+}^{T}$ and $\phi$. 
from the slopes of the straight lines obtained by plotting $\log i_{+} T^{\frac{1}{2}}$ instead of $\log N_{+} T^{\frac{1}{2}}$ against. $1 / T$ as $7.07 \pm 0.08 \mathrm{eV}$ for tungsten at the temperature range $1636^{\circ}$ to $1826^{\circ} \mathrm{K}$ and as $6.73 \pm 0.07 \mathrm{eV}$ for rhenium at the range $1636^{\circ}$ to 1778 ${ }^{\circ} K$, respectively. These results are shown in Fig. 2. It may be considered that these results are influenced by the extracting field of ions from the metallic surface, but it was found that the influence was very small. ${ }^{23)}$

Using a value of $5.69 \mathrm{~V}$ for the first ionization potential of strontium, the values of $\phi$ were calculated from Eq. (8) for tungsten and rhenium applied with strontium oxide as $2.73 \pm 0.22 \mathrm{~V}$ and $3.91 \pm 0.21 \mathrm{~V}$, respectively. In Table 1 the values of $l_{0}^{T}, l_{+}^{T}$ and $\phi$ are summarized.

In the studies of the thermoelectronic emission from the metallic surfaces applied with strontium oxide by the same manner as above, the values of $\phi$ were also estimated as $2.05 \pm 0.05 \mathrm{~V}$ for tungsten and as $2.99 \pm 0.05 \mathrm{~V}$ for rhenium, respectively. ${ }^{23)}$ Here, the agreement between both studies is poor. The differences may be caused by the fact that the ion emission occurs at the surface of the higher work function but the thermoelectronic emission at the lower.

Besides, in the studies with a method impinging the neutral atoms on clean metallic surfaces, the values of $4.58 \mathrm{~V}$ and $5.17 \mathrm{~V}$ have been reported for the work functions of tungsten and rhenium, respectively. ${ }^{24)}$ The values of $\phi$ reported above are in good agreement with those obtained by the measurements of the thermoelectronic emission from clean surfaces.

The ratio of the fluxes of strontium ions to atoms vaporized from strontium oxide on rhenium is given by,

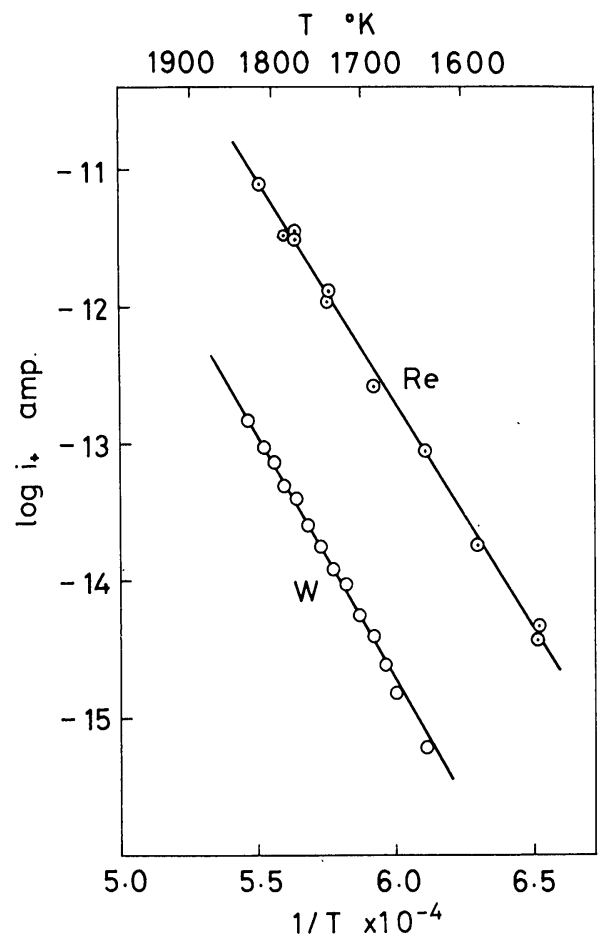

Fig. 3. $\log i_{+}$v.s. $l / T$.

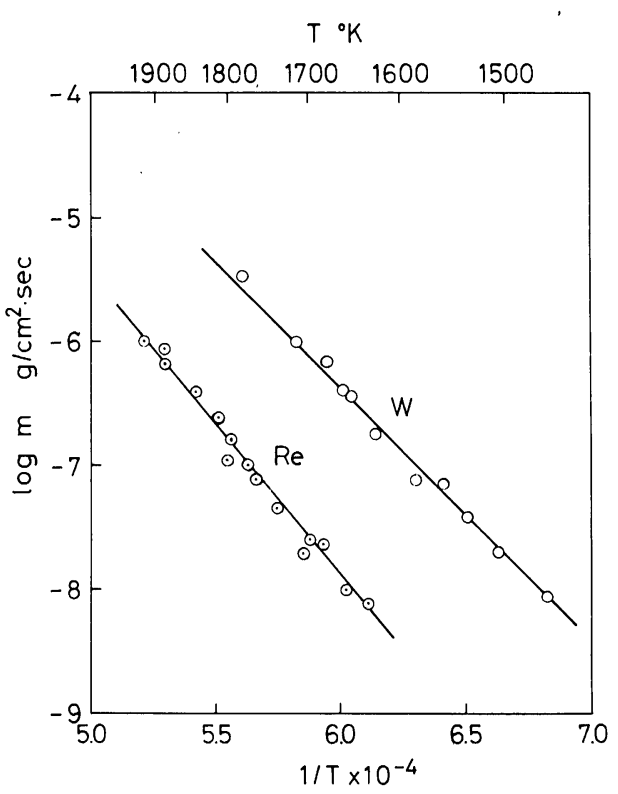

Fig. 4. Rate of vaporization of strontium atoms from strontium oxide applied on tungsten and rhenium. 


\section{Vaporization of Strontium Atom}

and Ion from Storontium Oxide

Table 2. Values of left hand of Eq. (14).

\begin{tabular}{ccccccc}
$T^{\circ} K$ & $\log i_{+\mathrm{Re}}$ & $\log i_{+\mathrm{W}}$ & $\log m_{\mathrm{W}}$ & $\log m_{\mathrm{Re}}$ & $2.303 k T$ & left hand of Eq. (14) \\
\hline 1600 & -13.50 & -15.58 & -6.85 & -8.48 & 0.318 & 1.18 \\
1700 & -12.32 & -14.30 & -6.15 & -7.59 & 0.337 & 1.15 \\
1800 & -11.25 & -13.16 & -5.48 & -6.79 & 0.357 & 1.15 \\
1900 & -10.50 & -12.09 & -4.87 & -6.03 & 0.377 & 1.11 \\
\hline
\end{tabular}

$$
\frac{N_{+\mathrm{Re}}}{N_{\mathrm{ORe}}}=\frac{\omega_{+}}{\omega_{\mathrm{0}}} \exp \left[\frac{\mathrm{e}\left(\phi_{\mathrm{Re}-\mathrm{SrO}}-I\right)}{k T}\right],(10)
$$

and on tungsten,

$$
\frac{N_{+W}}{N_{\mathrm{WW}}}=\frac{\omega_{+}}{\omega_{0}} \exp \left[\frac{\mathrm{e}\left(\phi_{\mathrm{W}-\mathrm{SrO}}-I\right)}{k T}\right] .
$$

Taking the ratio of the logarithmic forms of Eqs.(10) and (11), '

$$
\begin{aligned}
2.303 & {\left[\left(\log N_{+\mathrm{Re}}-\log N_{+W}\right)+\right.} \\
& \left.\left(\log N_{\mathrm{OW}}-\log N_{\mathrm{ORe}}\right)\right] k T \\
= & e\left(\phi_{\mathrm{Re}-\mathrm{SrO}}-\phi_{\mathrm{W}-\mathrm{SrO}}\right) .
\end{aligned}
$$

Using Eq.(9) and the following relation,

$$
N_{0} \propto m,
$$

Eq. (12) becomes,

$$
\begin{aligned}
& 2.303\left[\left(\log i_{+\mathrm{Re}}-\log i_{+\mathrm{W}}\right)+\right. \\
& \left.\left(\log m_{W}-\log m_{\mathrm{Re}}\right)\right] k T \\
& \quad=e\left(\phi_{\mathrm{Re}-\mathrm{SrO}}-\phi_{\mathrm{W}-\mathrm{SrO}}\right) .
\end{aligned}
$$

With the experimental data of $i_{+}$in Fig. 3 and $m$ in Fig. 4 , the values of the left hand side of Eq. (14) were calculated for the temperatures of $1600^{\circ}$, $1700^{\circ}, 1800^{\circ}$ and $1900^{\circ} \mathrm{K}$. These reusults are in good agreement with the value of the right hand side of Eq.(14), i.e., 1.18 $\pm 0.43 \mathrm{eV}$ as shown in Table 2 .

The values of $l_{0}^{T}$ obtained by many workers are in poor agreement with each other, but it could be concluded from the above discussions that the values of $l_{0}^{T}$ and $l_{+}^{T}$ estimated in the present work are very reasonable ones and the vaporization processes are described by the Saha-Langmuir equation.

\section{References}

1) J. Drowart and P. Goldfinger, "Advances in Mass Spectrometry, Vol.3" edited by W. L. Mead, pp.923

(1966), The Institute of Petroleum, London.

2 ) J. L. Margrave, "The Characterization of High Temperature Vapors"

(1967), John Wiley and Sons, New York.

3 ) M. Rutner, P. Goldfinger and J. P. Hirth, "Condensation and Evapora. tion" (1964), Gordon and Breach, New York.

4) B. Perević and D. Tošić, "Proceed. ings of the Seventh International Conference on Phenomena in Ionized Gases, Vol.1"(1966), Gradevinska Knjiga Pub. House, Beograd.

$5)$ A. N. Nesmeyanov, "Vapor Pressure of the Chemical Elements" (1963),

* The amount of strontium atom vaporized from strontium oxide is considerably more than 1000-fold greater than that of ion.3) Consequently, the amount of ion can be neglected in this relation. 


\section{M.Asano, K.Kubo and S.Magari}

Elsevier, Amsterdam.

6) C. Herring and M. H. Nichols, Rev. Modern Phys., 21, 185 (1949).

7 ) J. P. Blewett, J. Appl. Phys., 10. 668, 831 (1939).

8) A. Claassen and C. F. Veenemans, Z. eits. f. Phys., 80, 342 (1933).

$9)$ G. E. Mroore, H.W. Allison and J. D. Struthers, J. Chem. Phys., 18. 1572 (1950).

10) G. E. Moore, H. W. Allison and J. Morrison, ibid., 18, 1579 (1950).

11) A. H. White, J.Appl. Phys., 20, 856 (1949).

12) L. T. Aldrich, ibid., 22. 1168 (1951).

13) P. W. Bickel and L. V. Holroyd, J. Chem. Phys., 22, 1793 (1954).

14) R. F. Porter, W. A. Chupka and M. G. Inghram, ibid., 23. 1347 (1955).
15) M. Kaminsky, "Atomic and Ionic Impact Phenomena on Metal Surfaces," pp. 127 (1965), Springer, Berlin.

16) S. Magari and K. Kubo, Rev. Scient. Instru., 36, 403 (1965).

17) M. Asano, K. Kubo and S. Magari, To be published.

18) L. Langmuir, Phys. Rev. 2, 329 (1913).

19) L. Wohler and F.D. Rossini, Cited in reference (10).

20) M. D. Scheer and J. Fine, J. Chem. Phys., 42, 3645 (1965).

21) M. D. Scheer and J. Fine, ibid., 46. 3998 (1967).

22) M. D. Scheer and J. Fine, ibid., 47. 4267 (1967).

23) N. Iyemura, K. Kubo, M. Asano and S. Magari, Unpublished data.

24) J. R. Werning, UCRL-8455 (1958).

25) A. Eberhagen, Fortschr. Phys., 8, 245 (1960). 